\title{
Impacts of Launch Vehicle Fairing Size on Human Exploration Architectures
}

\author{
Sharon Jefferies \\ NASA Langley Research Center \\ Hampton, VA 23681 \\ 757-864-4248 \\ sharon.a.jefferies@nasa.gov
}

\author{
Tim Collins \\ NASA Langley Research Center \\ Hampton, VA 23681 \\ 757-864-3113 \\ timothy.j.collins@nasa.gov
}

\author{
Alicia Dwyer Cianciolo \\ NASA Langley Research Center \\ Hampton, VA 23681 \\ 816-434-5306 \\ alicia.m.dwyercianciolo@nasa.gov
}

\author{
Tara Polsgrove \\ NASA Marshall Space Flight Center \\ Huntsville, AL 35812 \\ 256-544-1274 \\ tara.polsgrove@nasa.gov
}

\begin{abstract}
Human missions to Mars, particularly to the Martian surface, are grand endeavors that place extensive demands on ground infrastructure, launch capabilities, and mission systems. The interplay of capabilities and limitations among these areas can have significant impacts on the costs and ability to conduct Mars missions and campaigns. From a mission and campaign perspective, decisions that affect element designs, including those based on launch vehicle and ground considerations, can create effects that ripple through all phases of the mission and have significant impact on the overall campaign. These effects result in impacts to element designs and performance, launch and surface manifesting, and mission operations.
\end{abstract}

In current Evolvable Mars Campaign concepts, the NASA Space Launch System (SLS) is the primary launch vehicle for delivering crew and payloads to cis-lunar space. SLS is currently developing an $\mathbf{8 . 4 m}$ diameter cargo fairing, with a planned upgrade to a $10 \mathrm{~m}$ diameter fairing in the future. Fairing diameter is a driving factor that impacts many aspects of system design, vehicle performance, and operational concepts. It creates a ripple effect that influences all aspects of a Mars mission, including: element designs, grounds operations, launch vehicle design, payload packaging on the lander, launch vehicle adapter design to meet structural launch requirements, control and thermal protection during entry and descent at Mars, landing stability, and surface operations.

Analyses have been performed in each of these areas to assess and, where possible, quantify the impacts of fairing diameter selection on all aspects of a Mars mission. Several potential impacts of launch fairing diameter selection are identified in each of these areas, along with changes to system designs that result. Solutions for addressing these impacts generally result in increased systems mass and propellant needs, which can further exacerbate packaging and flight challenges. This paper presents the results of the analyses performed, the potential changes to mission architectures and campaigns that result, and the general trends that are more broadly applicable to any element design or mission planning for human exploration.

\section{TABLE OF CONTENTS}

1. INTRODUCTION.. 1

2. ANALYSES AND RESULTS .................................... 2

3. OBSERVATIONS. 11
4. KEY FINDINGS.........................................................13

5. CONCLUSION ...............................................................14

ACKNOWLEDGEMENTS .........................................14

REFERENCES .......................................................14

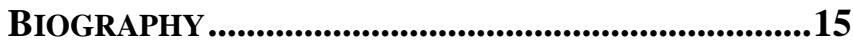

\section{INTRODUCTION}

Human missions to Mars, particularly to the Martian surface, are grand endeavors that place extensive demands on ground infrastructure, launch capabilities, and mission systems. The interplay of capabilities and limitations among these areas can have significant impacts on the costs and ability to conduct Mars missions and campaigns. From a mission and campaign perspective, decisions that affect element designs, including those based on launch vehicle and ground considerations, can create effects that ripple through all phases of the mission and have significant impact on the overall campaign. These effects result in impacts to element designs and performance, launch and surface manifesting, and mission operations.

This paper presents the results of a study conducted within NASA's Evolvable Mars Campaign (EMC) to evaluate the impacts of launch vehicle fairing diameter on human exploration systems and campaigns. Through a series of analyses, the study team assessed many aspects of a human exploration mission, including element design and performance, ground operations, launch, entry and descent at Mars, landing, and surface operations to understand how fairing diameter selection can affect vehicle and payload designs, and campaign options and decisions.

Current Space Launch System (SLS) development plans envision the use of both $8.4 \mathrm{~m}(27.6 \mathrm{ft})$ and $10 \mathrm{~m}(32.8 \mathrm{ft})$ cargo fairings to support future mission needs. The objective of this study was to identify necessary changes and limitations that may be imposed on exploration systems and campaigns if only the $8.4 \mathrm{~m}$ diameter fairing is developed, and the potential costs and limitations associated with adopting a 
$10 \mathrm{~m}$ diameter fairing. Additionally, the study sought to determine if a $10 \mathrm{~m}$ diameter fairing is necessary to support human exploration missions, and if so, when the larger fairing would need to be introduced.

It is hoped that these analyses will serve to inform decision makers when making development decisions on future SLS upgrades, and that the results will have broader applicability by informing mission and system developers both within and outside of NASA of the implications of, and challenges resulting from, launch fairing size on systems, operations, missions, and campaigns. The analyses in this study are based on the elements, manifests, and concepts of operations of the 'Point of Departure' (POD) architecture developed for the EMC [1], but the general observations and trends are potentially applicable to any human exploration mission to Mars.

\section{ANALYSES AND RESULTS}

\section{NASA Space Launch System Design}

Current SLS payload accommodation plans include evaluating the need for and size of both $8.4 \mathrm{~m}$ and $10 \mathrm{~m}$ diameter fairings (Figure 1) to support human exploration and science missions. Configuration (length, diameter) and availability (first need, cadence) decisions have implications for SLS development timelines and also vehicle capability and ground infrastructure requirements. Mission requirements in support of Mars campaigns are another factor affecting fairing configuration and availability decisions. If a campaign concept plans to incorporate a particular fairing size earlier than current planning, then a trade will be necessary to weigh SLS development budget against the impacts to the campaign implementation schedule.

Existing NASA Ground Systems Development and Operations Program (GSDO) infrastructure is capable of handling payload fairings up $19.2 \mathrm{~m}$ (63 ft) tall. A nearby government facility at Cape Canaveral Air Force Station (CCAFS) can accommodate up to $27.4 \mathrm{~m}$ (90 ft) tall fairings, but will require investment in infrastructure and/or transportation to support use on NASA missions. Potential mission needs and development costs are currently being investigated by NASA's Human Exploration Operations Mission Directorate (HEOMD). Since fairing length and payload center of gravity (CG) height have direct correlation to vehicle structural loading and launch performance, it is important for primary customers of SLS, like EMC, to help establish the best balance of payload diameter and length requirements as early as possible. This would help HEOMD understand what kind of missions might require an $8.4 \mathrm{~m}$ fairing or require a step up to a $10 \mathrm{~m}$ diameter fairing.

Adopting the $10 \mathrm{~m}$ fairing is dependent on performance upgrades for the Exploration Upper Stage (EUS) and replacing the current Solid Rocket Boosters used on SLS Block 1B with Evolved or Advanced Boosters. In order to support SLS Block 2 payloads and a 10m fairing, SLS will most likely require some structural modifications to the Core

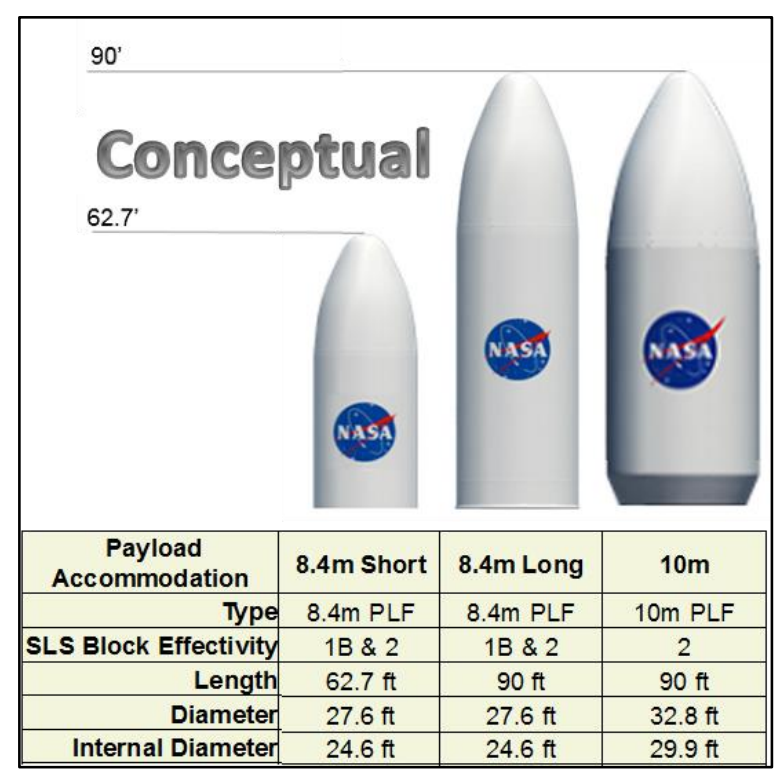

Figure 1. SLS fairing concepts for exploration missions.

Stage and EUS. This will be a fundamental upgrade in SLS capabilities and has an associated development cost. Before such an investment in the vehicle is made, HEOMD will study system impacts (technical, risk, funding and schedule) of different Mars components that can be designed to fit in either $8.4 \mathrm{~m}$ or $10 \mathrm{~m}$ fairings along with the impacts of potential vehicle changes. Determining when to make such an investment will be important both for managing SLS development costs and enabling missions reliant on the larger fairing. As with the $8.4 \mathrm{~m}$ fairing, the planned dimensions of the $10 \mathrm{~m}$ fairing are also constrained by fitting within, and through the door of the encapsulation facility. Currently this CCAFS government facility is the only one in the world that can accommodate a $27.4 \mathrm{~m}$ tall $10 \mathrm{~m}$ diameter fairing, and those resources must serve other customers in addition to NASA. This facility requires careful scheduling of launch stack integration and encapsulation activities across a wide user base and can take anywhere from one to six months to perform. Therefore, early definition of SLS fairing configuration and availability requirements will support costeffective planning and evolution of the SLS to meet Mars campaign needs.

\section{Payload Ground Handling and Testing}

The decision to use larger diameter elements, such as the Lander and Mars Ascent Vehicle (MAV), may be necessary for both launch and operations at Mars, but the challenges and costs associated with ground-based operations for these larger elements must be considered. As seen with SLS, existing infrastructure and facilities have limitations on the size of payloads that they can accommodate. A Constellationera study investigated ground transportation, testing, and processing considerations for proposed Altair lander configurations, which have dimensions similar to the Mars Lander and MAV. [2] 
Based on the study's survey of transportation capabilities, which looked at various transportation options, routes, transit times, and limitations, the only currently available method of transporting large $8-10 \mathrm{~m}$ elements long distances is by water. These routes can take several weeks between each intermediate destination, require several transfers involving critical lifts, and are subject to severe weather in all seasons (Figure 2). Some air options may become available with additional investment, such as new large-freight-capable airplanes or a new payload carrier for use with the Shuttle Carrier Aircraft, but the costs to develop, obtain, and maintain these systems can cost millions of dollars per year. Long-range land transport will continue to be restricted by federal and state regulations as well as road, rail, and bridge limitations.

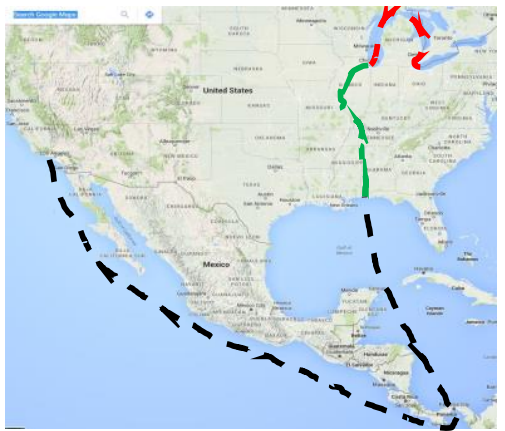

a)

Figure 2. Waterway routes a) Southern California to

Plum Brook Station and b) Plum Brook Station to KSC. Map data (2016 Google, INEGI. Legend: River routes green, lake routes red, and ocean routes black.

In addition to transportation challenges, the Constellation study also considered several challenges and gaps associated with test and integration capabilities for large, complex payloads. NASA's Plum Brook Station in Ohio has been designed to accommodate large spacecraft for various integrated environmental tests, but $8-10 \mathrm{~m}$ payloads exceed some test stand capabilities or will require special handling and test modifications to run. Similarly, payload processing facilities near the Kennedy Space Center launch site are not configured to simultaneously accommodate all of the hazardous processing operations needed to prepare largediameter payloads for launch.

Options for mitigating test and processing issues include designing smaller spacecraft, manufacturing near the launch site, waiving some or all of the integrated environmental testing, and testing lower levels of assembly but waiving fully-integrated element tests. Another alternative includes investment to build or upgrade facilities that can accommodate testing and processing needs for large diameter spacecraft. This option will include not only the cost of the initial build, but also the long-term maintenance and personnel costs to run and maintain the facility.

\section{Lander and Mars Ascent Vehicle Design}

As previously stated, this study used EMC architectures under development in FY2016 for the assessment. Within these architectures, only the Lander, MAV, and Mars aeroentry device concepts were based on the availability of a $10 \mathrm{~m}$ fairing, and modification of these vehicles to fit within an $8.4 \mathrm{~m}$ fairing poses some significant design impacts. This section discusses the Lander and MAV design impacts, and the aeroentry devices are covered in a later section.

The impacts are primarily driven by the need to package the large propellant tanks, engines, and landing gear within a narrower footprint. The only means of accomplishing this without breaking apart the vehicle and assembling in space or on the surface of Mars is to make the Lander and MAV taller. This results in a taller Lander, taller MAV, and taller overall payload stack, with higher component and overall CGs (Figure 3). Stretching the propellant tanks in this manner has the additional effects of increasing the tank mass and mounting complexity. A circular effect is observed with these modifications. The design changes result in a net increase in structure mass (Figure 4), which requires more propellant to land on the surface that, in turn, requires larger (taller) lander propellant tanks that further increase the height of the integrated vehicle.

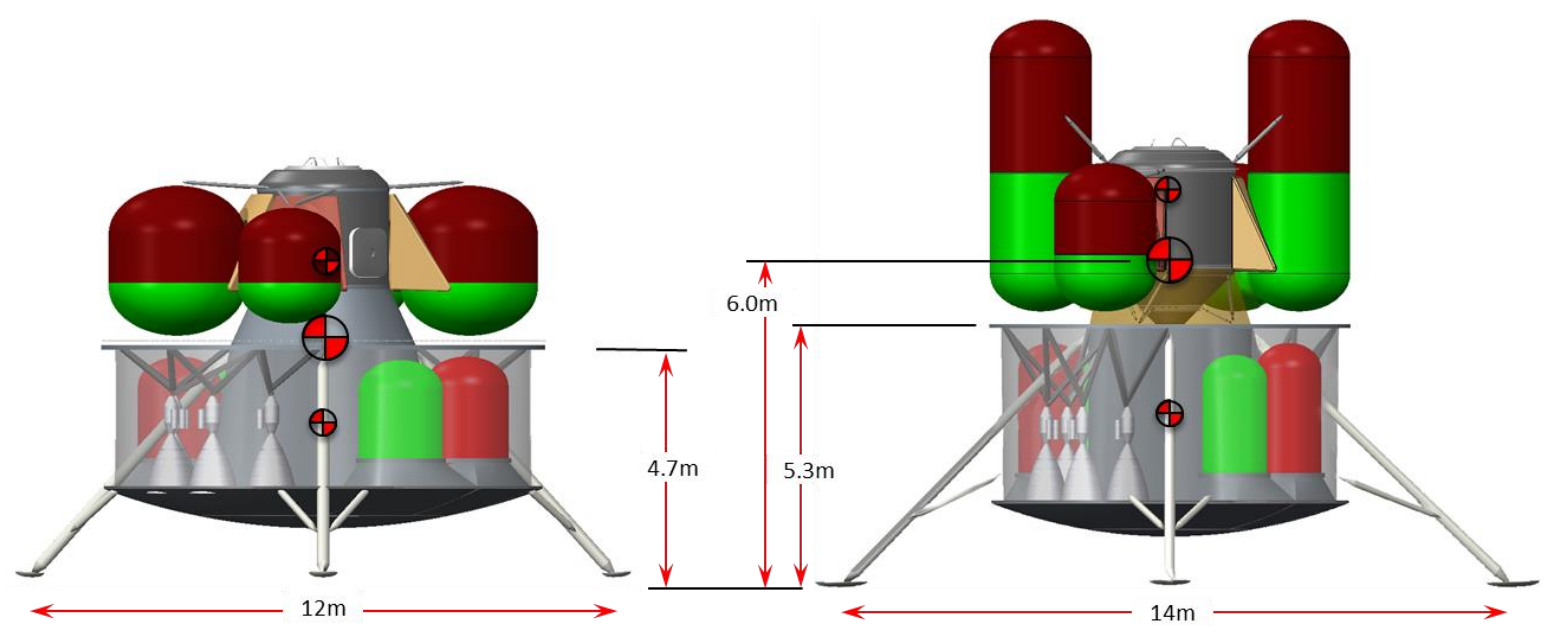

Figure 3. Representative $9.1 \mathrm{~m}$ (left) and $7.5 \mathrm{~m}$ (right) landers for respective $8.4 \mathrm{~m}$ and $10 \mathrm{~m}$ fairing diameters. 


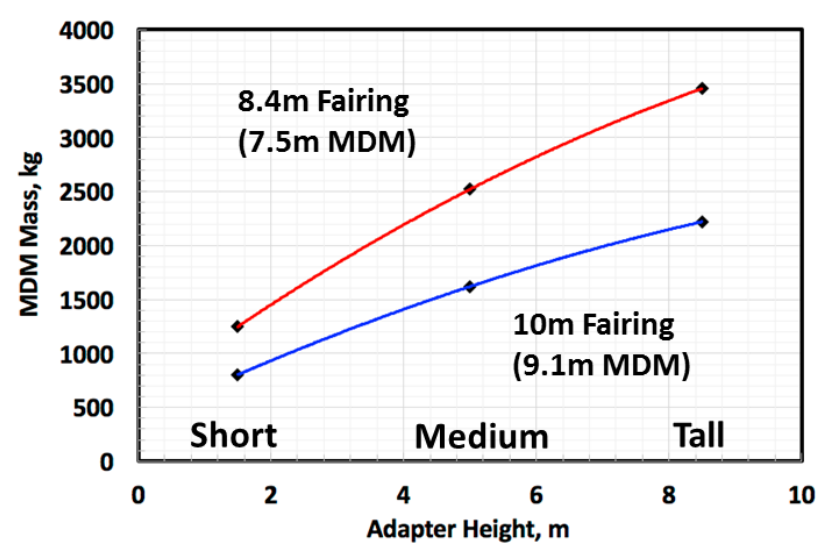

Figure 4. Comparison of Lander (MDM) mass for both fairing diameters at the short, medium, and tall stack heights.

The design changes also result in narrower (and possibly more angled) adapters between the Lander and launchvehicle core stage. These changes in adapter geometry result in less efficient load paths that decrease bending stiffness and produce lower modal (vibration) frequencies. The net effect is that more mass is required to meet structural performance requirements. These mass increases will be discussed in more detail later in the paper.

\section{Lander Packaging}

An assumption for this study is that the mission manifest (number of crew, in space transportation, parking orbits, etc.) could not change to accommodate the $8.4 \mathrm{~m}$ fairing. Therefore, a performance equivalent MAV and Lander must be rearranged, without reducing capability. For example, the solution could not consider taking a smaller less capable MAV.

When considering lander packaging, the team used volumetrically representative element models and attempted to find packaging configuration solutions for desired surface manifests. The surface manifests were provided by the EMC Mars surface team and represented the sequencing of capabilities desired on the surface for Mars crewed missions, balanced with lander payload capabilities, for the POD architecture. Packaging configurations using these manifests were generated for both $9.1 \mathrm{~m}$ and $7.5 \mathrm{~m}$ lander variants (dynamic/useable envelope for the $10 \mathrm{~m}$ and $8.4 \mathrm{~m}$ diameter fairings respectively). Lander packaging configurations were worked iteratively with the surface team, and both manifests and element design concepts were modified based on observations of the layouts. To minimize costs, EMC used a common Lander design with a landed capacity of approximately 20t to accommodate both the MAV and all other payloads. The initial manifests require four of the landers to deliver payload to support a surface mission.

For example, initial manifests included a $5 \mathrm{~m}$ diameter logistics module. Attempts to package this module with other payloads resulted in very tall and untenably-stacked payload configurations on both $7.5 \mathrm{~m}$ and $9.1 \mathrm{~m}$ landers. After observing the challenges with packaging this element, the logistics modules were redesigned to a smaller form factor that enabled packaging of one module below the lander deck in the well designed for the MAV ascent engines. This space is otherwise unused on landers that do not carry a MAV. While packaging payloads in the well requires additional integration mass, and the amount of logistics that can be carried in the module is reduced, these are acceptable costs when compared against the benefits of a lower overall CG and increased available deck area for other payloads.

Several challenges were identified when attempting to package the elements on the landers designed to fit in the $8.4 \mathrm{~m}$ fairing. Some of these challenges are with the packaging of payloads on the $7.5 \mathrm{~m}$ lander itself, such as: volume limitations that prevented packaging of some desired surface manifests, thus potentially delaying delivery of mission capabilities; off-nominal packaging necessary for some payloads, which may require redesign of the payload; tighter packaging of payloads; and reduced flexibility to position payloads to manage CG. A comparison of similar manifests packaged on each lander is shown in Figure 5.

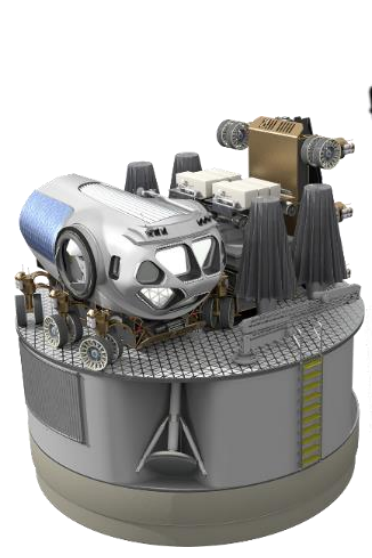

9.1m diameter lander

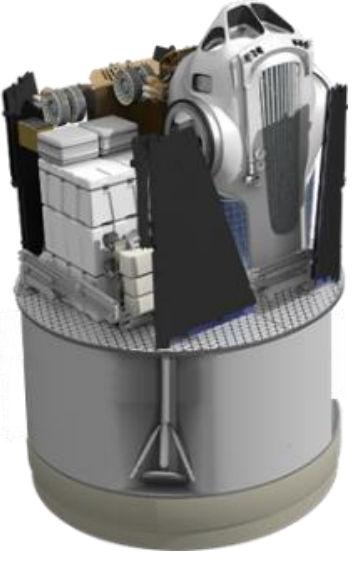

$7.5 \mathrm{~m}$ diameter lander
Figure 5. Side-by-side comparison of payload packaging for $9.1 \mathrm{~m}$ and $7.5 \mathrm{~m}$ diameter landers (all payloads common except surface power).

Additional challenges factor into other aspects of operations and performance. For instance, the taller overall payload stack height and CG location that result create challenges for meeting launch stack stiffness requirements, flight profile changes to mitigate flow impingement during entry and descent, landing gear design, and touchdown requirements to prevent tip-over. These are magnified as the lander height and CG location increase as a result of the narrower fairing diameter. Tighter packaging could pose challenges for offloading and may affect payload thermal management during transit. Limited deck space could restrict deployment of systems and operation of deck-mounted offloading devices.

The lander packaging layouts and CG estimates were used to inform several analyses, including launch stack packaging 
and adapter sizing, entry flight dynamics, entry heating and flow impingement, and surface offload sequencing and timeline development. Quick estimation of the center of gravity for each of the payload layouts was conducted using volumetrically representative CAD models with point masses reflecting current mass estimates and/or allocations from element MELs. Payload positions were adjusted to place CG in the approximate center of the lander deck.

It should be noted that, while the packaging layouts provided insights to potential payload integration structure needs, development of actual integration structure concepts and associated mass estimates were not completed as part of this study. Payload configurations that are taller, have offnominally oriented payloads, and/or payloads stacked on top of others are expected to require increased payload integration structural mass to withstand launch, entry, and landing loads.

\section{Launch Stack Analysis}

A key consideration for all mission manifests is the packaging and configuration of all required spacecraft components within the launch vehicle fairing. It is clear that smaller fairing diameters will require tighter packaging and taller stack configurations. It isn't feasible (at this time) to examine all the possible Mars launch configurations because of the wide variety of mission architectures that are still under consideration. However, for this study, a relatively difficult (tall) configuration was selected as representative and was analyzed in detail to investigate the implications of a smaller fairing diameter. The configuration selected consists of a MAV, Mars Descent Module (MDM), Hypersonic Inflatable Aerodynamic Decelerator (HIAD), Solar Electric Propulsion Module (SEP), and a Launch Vehicle Adapter (LVA). Additionally, there are smaller adapters that integrate the MAV and the SEP to the MDM. These configurations are shown (in cross section) in Figure 6 for both $10 \mathrm{~m}$ and $8.4 \mathrm{~m}$ fairings.

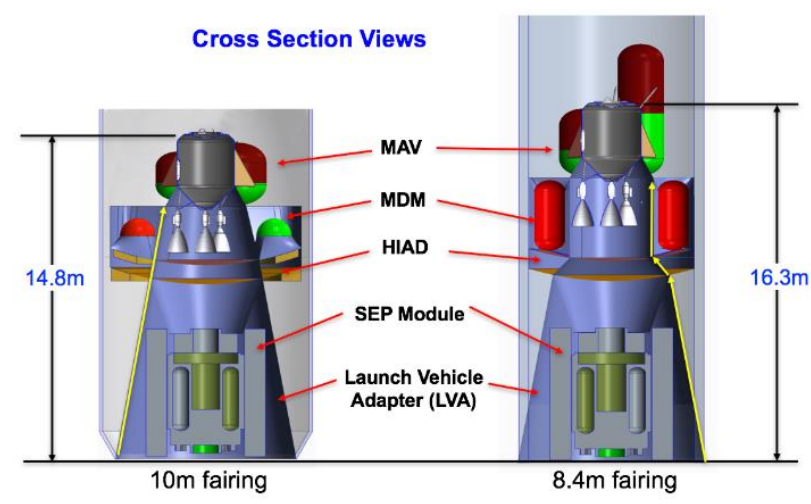

Figure 6. Cross section views of MAV/MDM/HIAD/ SEP launch stack configurations.

Both CAD and Finite-Element models were created for the stack configurations shown. These configurations consist of multiple large-mass payload elements stacked in sequence and interconnected by a series of adapters. There are few precedents for configurations of this class. Most historical payload configurations consist of relatively short stacks and short launch vehicle adapters. These stacks generally consist of a single payload element and single adapter that connects the payload to the launch-vehicle core stage. In these cases, it is typical to expect that mass will be driven by strength or possibly buckling failures of the primary structure. However, for taller stacks that consist of multiple payload elements and multiple adapters, the possibility (or even likelihood) exists that mass will be driven by stiffness (modal frequency). Smaller diameter stacks will, in general, have less stiffness than larger diameter stacks. Taller and smaller diameter stacks tend to result in non-optimal loads paths (long and not straight). The different load paths for the study case are shown in yellow in Figure 6.

In order to illustrate the possible impact of both diameter and height on mass, additional (shorter) models were created for purpose of comparison. These "medium" and "short" models are shown on the right side of Figure 7 with the nominal "tall" configuration on the left.

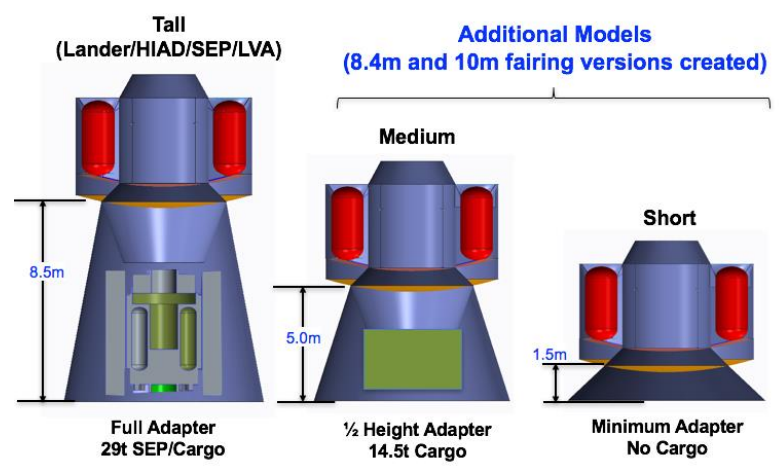

Figure 7. Analysis models for $8.4 \mathrm{~m}$ and $10 \mathrm{~m}$ fairing diameters.

The tall and short configurations are representative of specific Mars missions being considered by NASA. For the tall configuration the SEP is launched with the lander and for the short configuration it is launched via a separate launch vehicle. The Medium configuration is notional and might represent the inclusion of a secondary payload with the lander. The Tall, Medium, and Short configurations were analyzed for both the $8.4 \mathrm{~m}$ and $10 \mathrm{~m}$ fairing diameters $(7.5 \mathrm{~m}$ and $9.1 \mathrm{~m}$ lander diameters). Thus, a total of six analysis cases were run (only the $8.4 \mathrm{~m}$ models are shown in Figure 7), which allowed for the generation of representative sensitivity curves.

For each of the six models, failure modes related to strength, buckling, and stiffness (frequency) were considered. Although a firm frequency requirement has not been set for NASA's heaviest-lift SLS vehicles, for this (and other) studies a target lateral frequency of $5 \mathrm{~Hz}$ has been selected as a reasonable assumption. This is a relatively nonconservative frequency when compared to specifications in most commercial launch vehicle planning guides. The finite element analyses included $5 \mathrm{~g}$ axial and $2 \mathrm{~g}$ lateral launch loads. Structural components were constructed from 
composite sandwich structure with quasi-isotropic face sheets. The results obtained by analyzing the three stack heights for $8.4 \mathrm{~m}$ and $10 \mathrm{~m}$ fairing diameters are shown in Figure 8 and Figure 9.

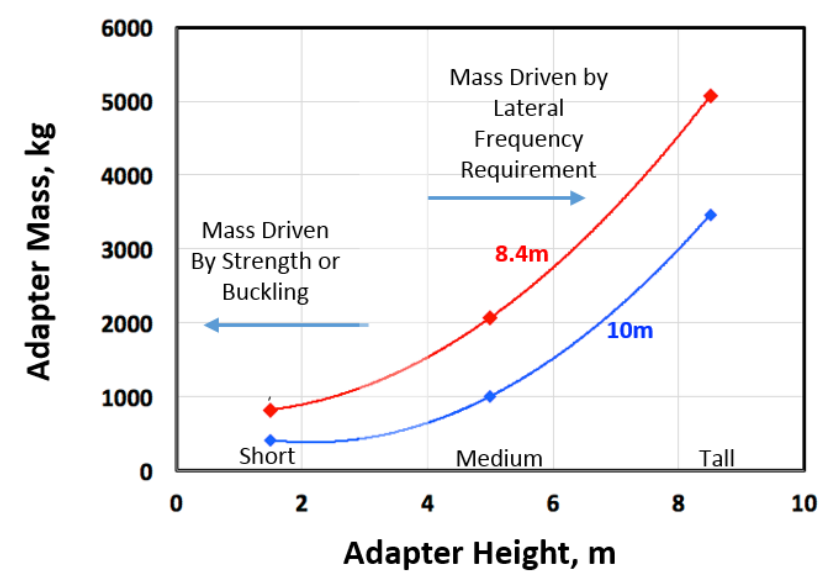

Figure 8. Impact of stack height (adapter height) on launch vehicle adapter mass.

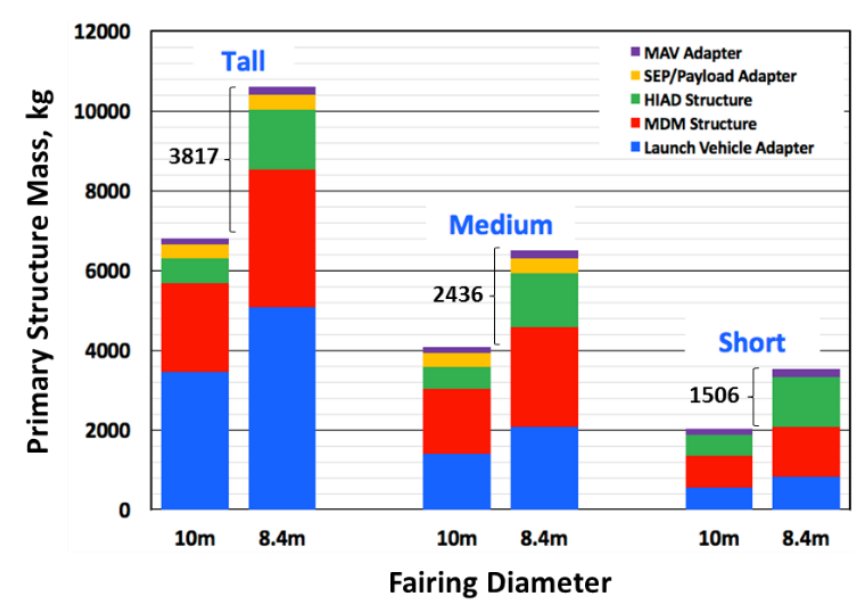

Figure 9. Impact of stack height on element structures mass for short. medium. and tall stack heights.

Figure 8 shows the mass of the primary launch vehicle adapter (LVA) for each case analyzed. For the short stack cases, the adapter mass is driven by strength and buckling as expected. For the medium and tall stacks, the adapter mass is driven by the assumed $5 \mathrm{~Hz}$ lateral frequency requirement. The differences are exacerbated for the smaller diameter fairing, which inherently results in less efficient loads paths and lower lateral stiffness. The difference between the LVA masses for the smaller and larger diameters is approximately $1.5 \mathrm{t}$ for the tall stack case.

The implications of lateral frequency and smaller diameter are not limited to the primary LVA. Other structural components in the stack are also affected. Figure 9 clearly shows the mass increase when decreasing fairing diameter to $8.4 \mathrm{~m}$ and the sensitivity of both the MDM and HIAD structural masses to diameter and stack height.
For the taller cases, both the MDM and HIAD structures require additional stiffening (and mass) to meet the assumed $5 \mathrm{~Hz}$ lateral frequency requirement. For the assumptions made here, the MDM mass difference due to diameter alone (short case) is approximately $1 \mathrm{t}$. The MDM mass increases to nearly $3 \mathrm{t}$ when both smaller diameter and increased height are considered.

It is emphasized that the purpose of the results shown is to illustrate trends and sensitivities (not to produce optimized or final structural designs). The authors believe the general trends are representative for most "tall" stack configurations, and thus, so are the implications related to diameter and stiffness. Ultimately, coupled-loads analyses are required to assess stack stiffness issues and perhaps tall stacks with low stiffness can be accommodated by vehicle control alone. However, reasonable frequency targets are typically part of the planning process for payload integration. Other mitigations could include tailored composite layups (plies biased to increase stiffness), or higher stiffness materials (M55J versus IM7 composite fibers for example). Additionally, for tall stacks in the class examined here, it may be necessary to consider attachment of the payload stack to the launch vehicle fairing in order to increase stiffness. It should be noted that for the tallest stacks analyzed, even small increases in stiffness (frequency) require a large amount of additional structural mass. For some stacks it may not be possible to achieve a desirable frequency target.

The key observation of the launch stack analysis is that payload integration mass may depend significantly on height and diameter, as well as payload stiffness requirements. Required stack elements and their arrangement/integration should be an integral part of both the mission planning and fairing design processes. The trends shown are representative of the issues and concerns related to diameter, height, and stiffness across all payload stack arrangements.

\section{Entry and Descent at Mars}

As mentioned previously, the assumption for this study is that a performance-equivalent MAV and lander must be rearranged, without reducing capability. Therefore, the descent module, payload, and entry system are allowed to grow in one dimension, axially, so that the vehicle gets taller or longer. This option has system level impacts for Entry, Descent, and Landing (EDL) on Mars. This section describes EDL system designed to accommodate the $10 \mathrm{~m}$ packaging arrangement and the system level changes that have to be made to package the same payload elements in an $8.4 \mathrm{~m}$ diameter fairing.

For this study, three entry technologies are considered. Each integrates with the payload and descent stage in a slightly different manner. Yet all concepts were initially designed to carry an equivalent cargo element, the Mars Ascent Vehicle, in a $10 \mathrm{~m}$ fairing. 
The three entry concepts include two low lift-to-drag (L/D) ratio, low ballistic number vehicles including the Adaptive Deployable Entry and Placement Technology (ADEPT) [3] and the Hypersonic Inflatable Aerodynamic Decelerator (HIAD) [4,5]. For these entry systems payload elements are arranged vertically in the launch vehicle. The ADEPT vehicle uses an umbrella-like rigid rib structure that supports a flexible TPS material with a $70^{\circ}$ sphere cone shape. Likewise, the HIAD vehicle uses a series of inflated tori to create a $70^{\circ}$ sphere cone shape that are covered with flexible TPS. A third vehicle, called the Mid L/D [6], uses a horizontal packaging

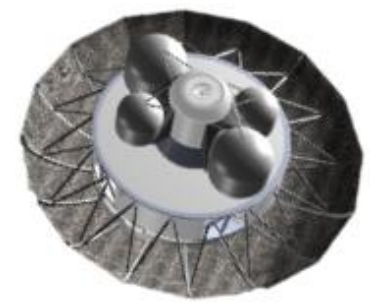

(a) ADEPT

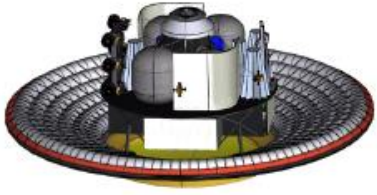

(b) HIAD

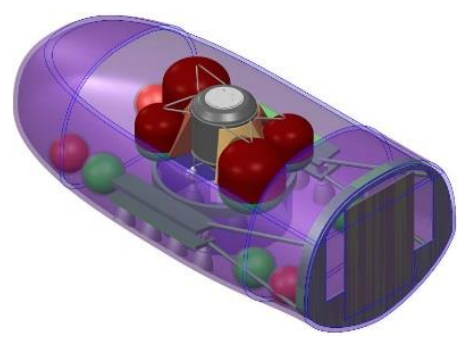

(c)Rigid Mid L/D

Figure 10. EMC MAV payload integrated with various entry technologies.

arrangement. The three entry system configurations integrated with the descent stage and the MAV payload are shown in Figure 10. Figure 11 shows the arrangement of the vehicles in the $10 \mathrm{~m}$ launch fairing. These specific point designs to deliver the 20t MAV payload to the surface of Mars are part of a larger EDL study that is summarized in Reference 7 . They provide a point of departure for the fairing diameter study.

Launch and landing phases of flight require the CG to be located on the long axis of symmetry. However, for EDL to achieve the desired guided aerocapture and entry performance, the $\mathrm{CG}$ is deliberately offset to generate the desired lift to drag ratio. For example, Mars Science Laboratory was designed to fly with a trimmed angle of attack corresponding to a $\mathrm{L} / \mathrm{D}$ of 0.24 , which required a $\mathrm{CG}$ offset that was generated by jettisoning $150 \mathrm{~kg}$ prior to entry. Then during entry, the reaction control system was used to control the lift vector by rotating the bank angle such that the vehicle reduced the down- and cross-range errors prior to parachute deployment. One disadvantage of using bank angle control is that during the bank angle reversals the vehicle spends a portion of the flight in which the vehicle is not controlled and targeting errors grow. Also, the approach of jettisoning mass to achieve the desired CG location is not

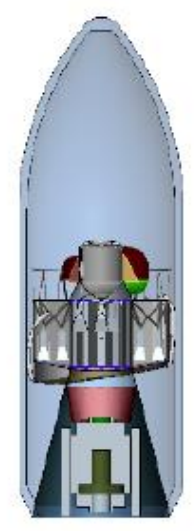

(a) ADEPT
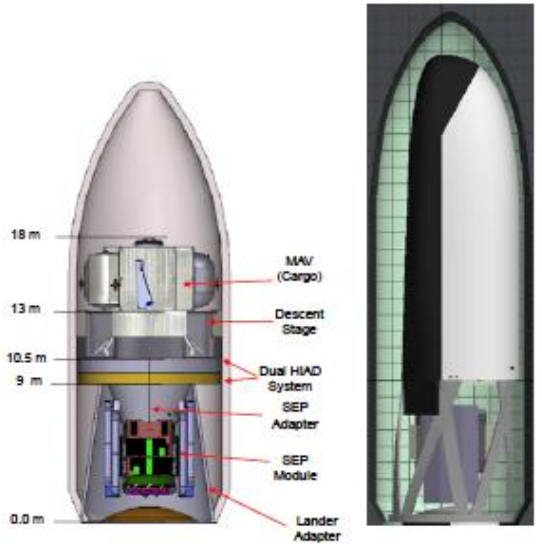

(c) Mid L/D
Figure 11. Launch vehicle stack configuration for (a) ADEPT, (b) HIAD and (c) Mid L/D.

feasible for large human-scale payloads so alternate approaches are being considered.

Figure 12 shows the trim lines (the $\mathrm{CG}$ location to achieve an $\mathrm{L} / \mathrm{D}=0.33$ ) for different angles of attack for a $23 \mathrm{~m}$ diameter $70^{\circ}$ sphere cone low-L/D vehicle. Note that steeper angles of attack require $\mathrm{CG}$ locations farther from the centerline. Also, as CG location moves closer to the nose ( $0,0 \mathrm{~m}$ location), it will need to be farther from the center line than if the $C G$ is located farther aft. A general rule of thumb used to ensure vehicle stability is to require the CG location to be below a point defined as the ratio of the distance aft from the nose (x) to the diameter to be less than or equal to 0.35 . In Figure 12 that location would be about $-8 \mathrm{~m}$ on the $\mathrm{x}$-axis.

This study considered a slightly smaller diameter Low L/D vehicle, $16 \mathrm{~m}$ instead of the $23 \mathrm{~m}$ diameter shown in Figure 12. For a particular lander manifest that contained a pressurized rover and logistics module that was originally packaged in a

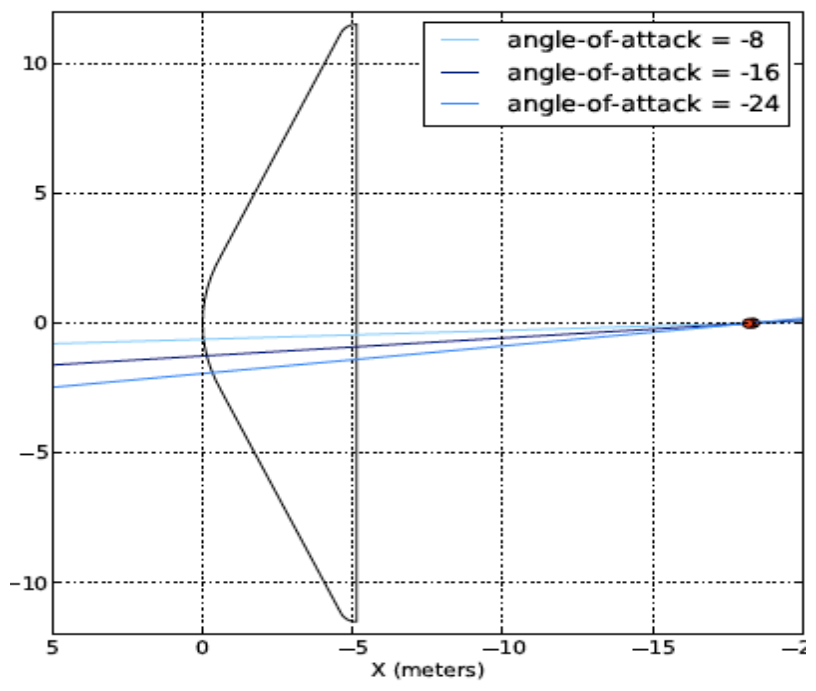

Figure 12. Low $L / D(L / D=0.3)$ vehicle trim lines at Mach 18 for various angles of attack. 
$10 \mathrm{~m}$ fairing (9.1m dynamic envelope) the $\mathrm{x}$-axis cg location was at $-4.6 \mathrm{~m}$. When the same payload elements were repackaged in the $8.4 \mathrm{~m}$ diameter launch vehicle $(7.5 \mathrm{~m}$ dynamic envelope) the stack height increased and the $\mathrm{CG}$ location was raised to $-5.3 \mathrm{~m}$. For the $16 \mathrm{~m}$ diameter HIAD, the stability limit based on the defined rule, is $-5.6 \mathrm{~m}$. The $8.4 \mathrm{~m}$ vehicle packaging has much less margin to the CG instability limit.

Another impact of the higher payload stack that results from rearranging the payload to accommodate the $8.4 \mathrm{~m}$ fairing is the threat of flow impingement on the payload. To reduce system mass, the Low L/D HIAD and ADEPT configurations do not have a backshell to protect the payload. Figure 13 shows the effect of flow impingement on a payload for two different angles of attack.

The impact of flow impingement on the payload is less for lower angles of attack. Therefore, it is desirable for the EDL system to fly at the minimum angle of attack that meets the desired landing constraints. Several flight control options are being considered that minimize angle of attack [8]. However, other options exist to reduce the flow impingement. One option is to increase the decelerator (HIAD or ADEPT) diameter, but that also increases the mass of the system. Another option is to cover the payload with a rigid backshelllike structure, an option that is more massive than increasing the diameter of the decelerator. Still another option is to reduce the height of the payload by offloading payload elements to additional landers, which also adds launches and increases the cost of the overall mission manifest.

There is an additional packaging consideration for the ADEPT vehicle. Some concepts of the umbrella-like deployable system package around the payload. This can create challenges to accessing the payload while in the launch vehicle and it also takes up some of the payload volume. Estimates indicate that stowing ADEPT around the payload may reduce the usable payload diameter in the $10 \mathrm{~m}$ launch fairing from $9.1 \mathrm{~m}$ to $8.6 \mathrm{~m}$. There is some amount of packaging margin within the $10 \mathrm{~m}$ fairing that that may reduce or eliminate the impact on payload packaging. However, if the fairing diameter is smaller, there may not be any available margin to use to avoid having to repackage or stack payload elements. Such is the case for the $8.4 \mathrm{~m}$ fairing, where ADEPT reduces the available payload diameter from $7.5 \mathrm{~m}$ to $\sim 7 \mathrm{~m}$. A
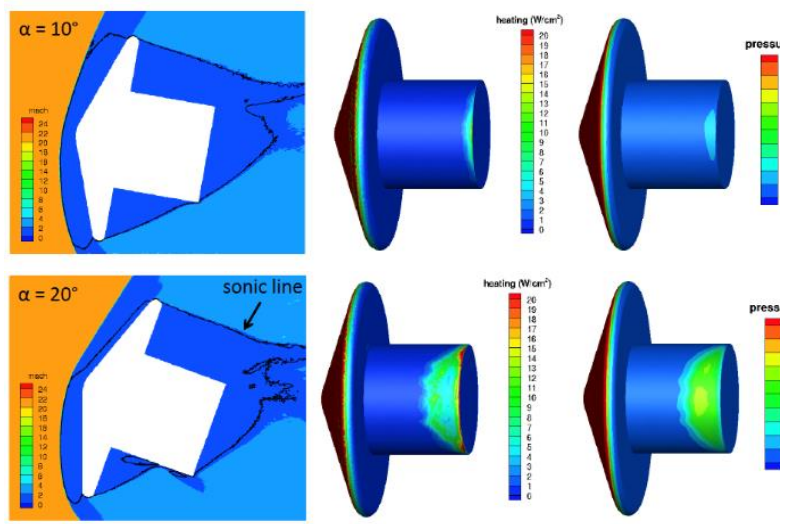

Figure 13. Sonic lines and relative heating and pressure for a $16 \mathrm{~m}$ diameter Low $\mathrm{L} / \mathrm{D}$ vehicle with a payload height of $9 \mathrm{~m}$ and diameter of $7.5 \mathrm{~m}$ for two different angle of attack.

full analysis of the repackaging effort for this case has not been performed. However, there has been an ADEPT concept developed that stows the deployable forward of payload (lower in the launch vehicle than that shown in Figure 11a).

As shown in Figure 10c and Figure 11c, the Mid L/D is another configuration being considered for EDL. The vehicle is designed to fit within the $9.1 \mathrm{~m}$ dynamic envelope. The SLS fairing protects the vehicle during the launch environment, then the vehicle is exposed for the duration of the mission, during which it protects the payload. The cross section of the vehicle shape is non-symmetric with a flattened bottom. This design choice results in an increased drag area which significantly improves entry performance at Mars. Analysis has shown adequate packaging volume inside the Mid L/D vehicle for currently sized payloads, given the $10 \mathrm{~m}$ fairing. The Mid L/D vehicle and its dimensions are shown in Figure 14.

Though a reduction to the $8.4 \mathrm{~m}$ launch vehicle fairing has not yet been studied, a few statements can be made about the impact to the current design, which would be significant. Simply scaling by length in all dimensions would result in $40 \%$ less volume and would not fit payloads as currently designed. Potential solutions include removing the fairing entirely and sizing the vehicle to the $8.4 \mathrm{~m}$ diameter, changing the fineness ratio of the vehicle to increase internal volume,
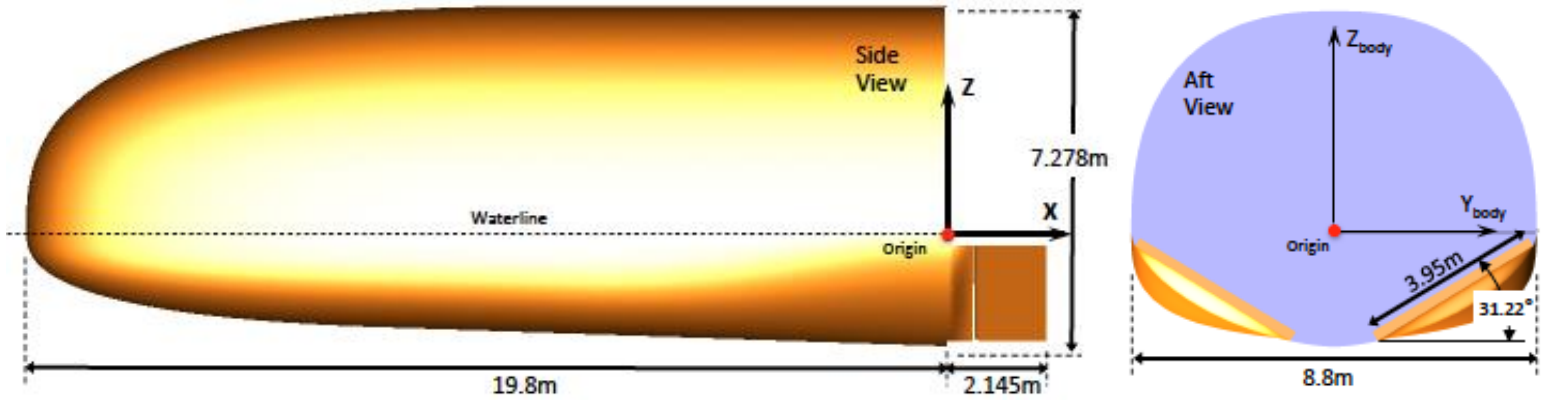

Figure 14. Side view (left) and end view (right) of the Mid L/D vehicle sized for the 10m SLS fairing. 
redesigning the payloads to better maximize use of the internal volume, a reduction in mission requirements, or some combination of the above.

Removing the fairing and exposing the Mid L/D to the ascent environment would result in a reduction in width from $8.8 \mathrm{~m}$ to $8.4 \mathrm{~m}$, assuming no portion of the vehicle can extend outside the circular cross-section. Scaling by length would result in the reduction of volume by $13 \%$. This amount of volume reduction could likely be dealt with by some combination of increasing the vehicle length and/or adjustments to payload and vehicle packaging. Alternatively, it may be possible to let the vehicle cross-section exceed the $8.4 \mathrm{~m}$ diameter as needed and create a fairing from the aft portion of the entry vehicle (near the flap, to the right in Figure 14) to blend the geometry to match the circular crosssection of the launch vehicle. Regardless, making the vehicle double as the fairing results in additional impacts to both the Mid L/D EDL vehicle and the launch vehicle. The Mid L/D vehicle must be designed to meet launch environment requirements, including acoustic loads which will require internal acoustic blanketing. The impact to the launch vehicle would include changes to the launch vehicle performance due to the change in aerodynamic shape, as well as additional considerations.

The second potential solution is to change the fineness ratio of the vehicle. In order to maintain the same volume, and assuming the width and height would be scaled down by 0.84 , the length would need to be scaled up by a factor of 1.42 , resulting in a length of $28 \mathrm{~m}(92 \mathrm{ft})$. This exceeds the current maximum fairing length as shown in Figure 1 and discussed in the SLS design section above. Additional mitigations (such as a change in Mid L/D shape, payload mass reduction, or payload redesign) may be able to reduce the length, but the vehicle is unlikely to fit in the $8.4 \mathrm{~m}$ short envelope even with mitigation, given current mission requirements and assumptions.

Regardless of launch fairing diameter, future work remains to characterize the entry control system, refine payload elements based on surface manifest assumptions, and perform detailed structural and packaging analysis for each design.

\section{Landing}

Landing gear design for human exploration-class Mars landers, with landed masses near or exceeding 20t, is expected to pose unique design challenges and risks. Landing gear design details are based on requirements to survive initial impact (possibly on a single leg), keep the deceleration rate below an acceptable limit, and prevent tip over. These requirements must be satisfied over a wide range of touchdown conditions that include velocities, ground slopes, soil friction, and other variables. Deceleration limits are typically achieved through use of an attenuation system (crushable material) built into each landing leg. The most well understood landing-gear design for a human lander is the design used by Apollo and Altair (the human lander concept of NASA's former Constellation Program). However, due to packaging constraints and increased landing loads (Mars landers have $\sim 40 \%$ more mass than Apollo), it is unlikely that Apollo-type landing gear will be suitable for Mars.

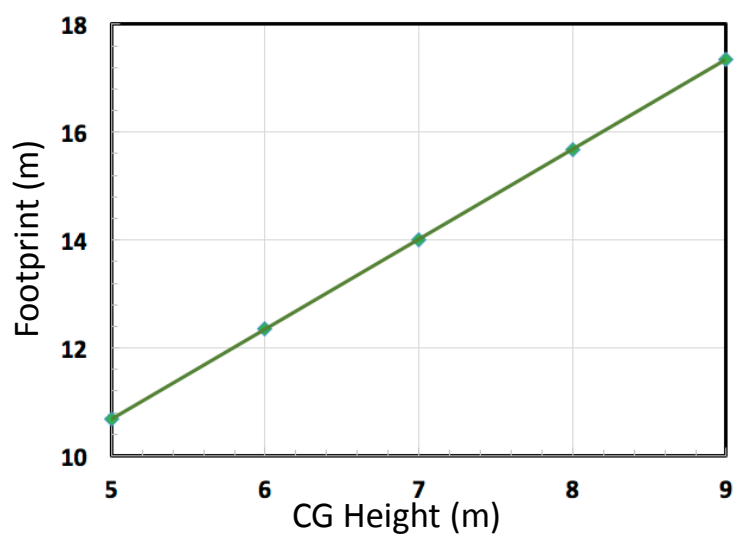

a) Footprint vs. CG height

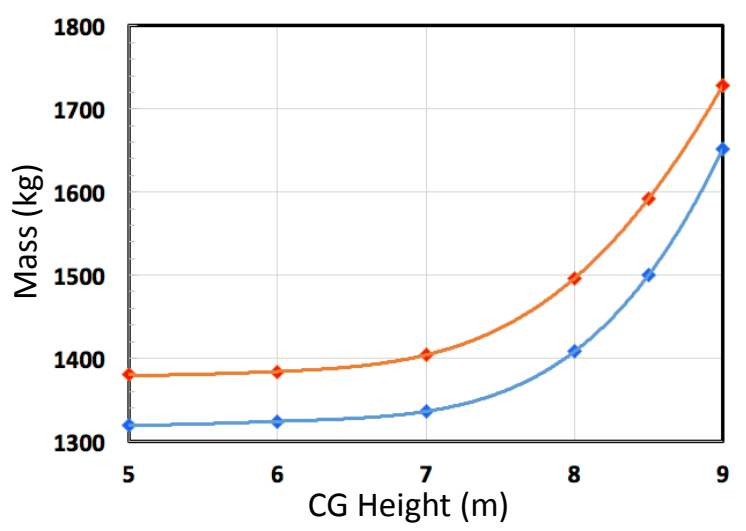

b) Landing gear mass vs. CG height

\section{Figure 15. a) Required stability footprint and b) mass (4 legs) for typical Apollo-type landing gear and 60t landed mass.}

For this study, existing analysis tools (Apollo/Altair based) were used to show the general impact that increased $C G$ height has on required landing gear footprint (spread between foot pads) and landing gear mass. The required footprint to prevent tip-over of an assumed 60t landed mass is shown in Figure 15a. These curves are based on a series of dynamic touchdown analyses (including tip-over) for a range of touchdown scenarios. The same dynamic models were used to bound the worst-case loads seen by any landing leg. These loads were used to generate mass versus CG height curves for both 9.1 and $7.5 \mathrm{~m}$ lander diameters (Figure 15b). As stated previously, although Apollo-style gear is likely not appropriate for Mars, the trends shown in the figures are expected to be representative across landing gear designs.

It can be seen in Figure 15b that for CG heights below $\sim 7 \mathrm{~m}$, the mass-growth curves are relatively flat. This is because for footprints below $\sim 14 \mathrm{~m}$ it is possible to optimize the orientation (angle) of each leg with respect to the lander descent module to minimize mass. For CG heights greater 
than $\sim 7 \mathrm{~m}$, landing gear mass increases exponentially. This is driven by non-optimal geometries and longer landing legs. As landing-leg length increases, the bending moment at touchdown increases, along with the tendency for buckling in the primary strut assemblies. To resist bending and buckling, the diameters (and mass) of the landing gear must be increased. In addition to increasing mass, more robust landing gear struts complicate the ability to package and deploy within limited space. Mass growth with increased CG height is exacerbated for the $7.5 \mathrm{~m}$ lander, which has both a higher CG and a need for longer legs to provide the necessary footprint.

The importance of the graphs Figure 15 are the trends shown and not the specific mass numbers. The figures indicate that for any given lander and launch vehicle scenario, it is important to establish the landed $\mathrm{CG}$ height, the required footprint, and whether or not the landing gear mass has moved into the region of exponential mass growth. For different landers, the transition CG height and the mass delta for different diameters may vary significantly. The figures also show that there will be landing-gear mass increase if the lander diameter is reduced from $9.1 \mathrm{~m}$ to $7.5 \mathrm{~m}$. The precise mass increase must be determined from detailed Mars landing gear design that includes the true geometry along with all struts, joints, and deployment mechanisms.

The attenuation system required for energy absorption and to maintain deceleration limits (g's) places additional limitations on the landing gear design. Landing must be highly controlled to protect crew and cargo during touchdown and to ensure a nearly upright orientation suitable for offloading and ascent. Both primary and secondary attenuators are likely required, placing further limitations on the landing gear geometry and packaging scheme. Engines and propellant tanks occupy most of the available Mars descent-stage volume, leaving little space for landing gear stowage. Packaging is difficult for the $9.1 \mathrm{~m}$ lander and even more so for the $7.5 \mathrm{~m}$ lander. For landers that retain their heat shield all the way to the surface, landing gear geometries will be further limited by the requirement to deploy through the heat shield surface.

In summary, the combination of leg length, strut diameter, and attenuation all contribute to the challenges of landing gear packaging and mass. Satisfying all requirements may result in a landing gear design that exceeds available volume or must protrude through the upper lander deck. These challenges increase with CG height and are exacerbated for smaller diameter landers.

\section{Surface Operations}

Deployment of systems, payload offloading, and access to the MAV and lander-mounted habitats are the surface operations expected to be most impacted by changes driven by fairing diameter selection. Tightly packaged landers and limited deck space leave little room for deployment and operation of systems, such as arrays, radiators, and offloading devices from the lander deck. Structure and mechanisms must support these deployed systems while allowing sufficient clearance for gaining access to the lander deck and for offloading payloads. This increases mass that must be accounted for in total payload mass and adds complexity and risk to the mission.

As an example, sizable radiators are needed for thermal management of lander-based atmospheric ISRU systems under consideration in the EMC. These radiators must be stowed in a manner that prevents contact with the MAV during launch and landing. Additionally, space is required for their deployment and operation, which typically means extending outward from the lander deck (Figure 16a). Structural mass must be added to support the radiators in this

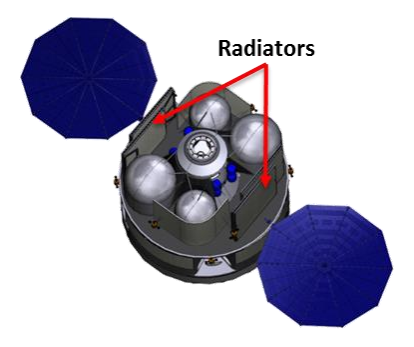

a) Stowed

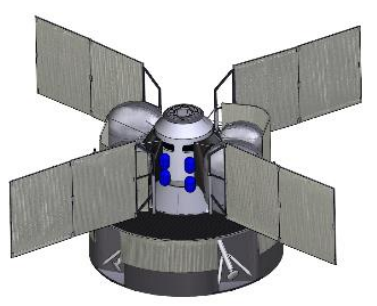

b) Deployed

\section{Figure 16. Radiators for MAV lander in a) stowed and b) deployed configurations.}

configuration.

In addition to having limited room to deploy, offloading devices also need room to operate to offload payloads. Close quarters on deck (Figure 17) may increase the probability of contact of the offloading device with payloads or other deployed systems, which is a safety and risk concern. Offloading operations may benefit from concepts to integrate payloads into more tightly packed stacks. However, those benefits may be eliminated if limited deck space requires stacking cargo on top of cargo. Reduced deck space also limits flexibility to optimize packaging and choreograph

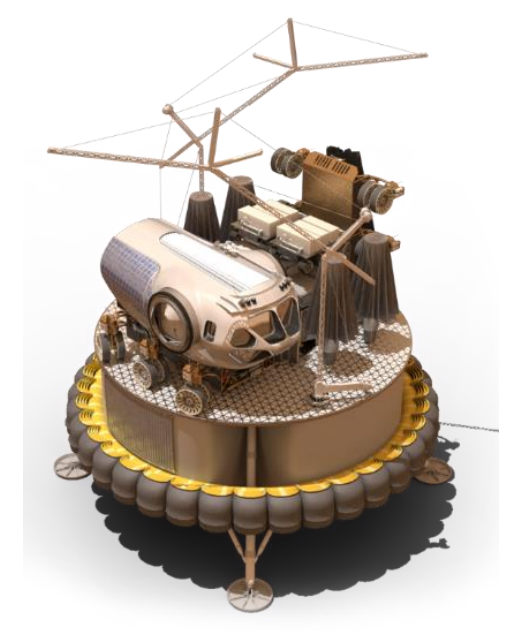

Figure 17. Representation of deployed offloading systems on crowded lander deck. 
autonomous offloading sequences.

Payload orientation for crew descent modules is another consideration. For some missions, the crew descends in a pressurized rover. Packaging limitations, particularly with the $7.5 \mathrm{~m}$ diameter lander, may require the rover to package in an off-nominal, vertical orientation. After descent, the crew will have to live in the rover in this orientation until the vehicle can be repositioned. Modifications to the rover design will be required to accommodate the crew while in this position. Reorienting the rover during offloading also adds risk to the operation, especially if the crew is on board.

Access to the MAV will be affected by overall lander deck height. Current concepts envision a tunnel for crew access to the MAV from a surface vehicle like a rover (Figure 18). Taller landers will increase the required length, and thus mass, of the tunnel. Steeper angles may increase the difficulty of climbing and carrying payloads up to the MAV, although this can be mitigated by adding a winch or similar device. Finally, the smaller diameter deck reduces the area available to the crew for packing and check-out of the MAV prior to departure.

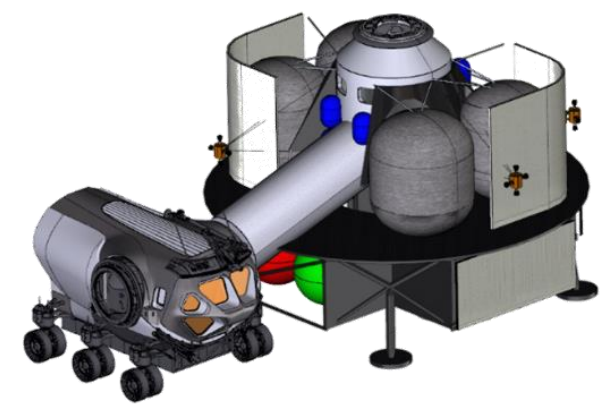

Figure 18. Mars ascent vehicle crew access configuration.

Lander deck height may also affect access to lander-mounted habitats. Within EMC, massive ( 20t) monolithic habitats delivered to the surface are not removed from the lander they descend with. Accomodations are necessary for crew access and to connect logistics and other modules. Tunnels with airlocks, ladders, and lifting devices to elevate and support mated modules are all potential solutions. The extra height of landers designed to fit in the $8.4 \mathrm{~m}$ diameter fairing adds to the mass and complexity of access solutions.

\section{OBSERVATIONS}

\section{Ripple Effect}

As seen by the analyses, many impacts of using the $8.4 \mathrm{~m}$ fairing size manifest as a mass increase, which must then be factored into launch mass. Often, a mass increase in one area produces a resultant mass increase in other areas. For example, as the MAV mass increases, the Lander may require additional propellant to safely land. Increases in Lander mass drive corresponding increases in launch vehicle adapter mass to meet stiffness requirements for the higher CG, and may increase in-space transportation system propellant needs. As launch masses grow, changes may be required to mission planning and campaign manifesting to ensure that payloads can be launched and delivered to the desired destination (Figure 19).

There are several campaign options for addressing increased launch mass, all of which will have implications individual on missions and campaigns. These options and their representative effects are captured in the following list. Trades will be required to understand

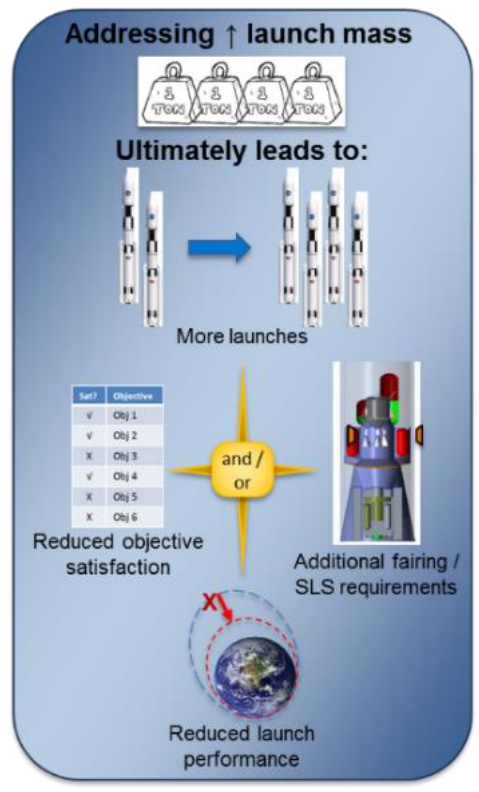

Figure 19. Potential campaign impacts of increasing launch mass. costs and benefits of each option for a given scenario.

- Launch systems separately: Dividing up launch stacks and launching elements separately can enable launch of fully integrated, unmodified systems. However, this option requires an additional launch and may require the addition of a boost stage or service module to support one of the elements, e.g., when launching the transit habitat separately from its in-space transportation stage. Further, these elements will have to be integrated in space to form the operational vehicle (e.g., Mars transit vehicle), and additional connection hardware mass may also be required.

- Offload elements and outfit/refuel in space: Some elements, particularly transportation stages and habitats, can be partially offloaded for launch. This typically means offloading propellant from transportation stages, which will require in-space refueling capabilities. For habitats, offloading can include logistics, spares, and some subsystems and components. The habitat must be designed to accommodate post-launch installation and outfitting of offloaded subsystems. Supply of offloaded fuel, logistics, and equipment may require development of special delivery elements, such as a fuel tanker, and additional launches.

- Remove elements from manifest: A decision could be made to not launch particular elements or lander payloads for a particular mission, reducing the launched mass and stack height. Opting to eliminate some systems from the mission, however, limits available mission capabilities and could lead to reductions in objective satisfaction for the mission. 
- Use fairing to support loads: Launch fairings could be designed to integrate with payload stacks to increase lateral stiffness and reduce adapter mass. However, this levies additional requirements on the fairing that are not currently included in development plans, and the increased fairing mass would affect all launches, even when load sharing is not required. Additional analysis is needed to determine if this option provides a net benefit across the campaign and whether other SLS design changes would also be required.

- Launch to lower altitude: A final option is to maintain the same launch stack, and launch to a lower initial apogee altitude. This option is discussed in the following section.

\section{Effect of Mass on Launch to Orbit}

One of the options identified for addressing increased system masses is to manifest the items as planned, but launch to a lower initial insertion orbit. As launched payload mass increases, the initial insertion apogee altitude that SLS can attain decreases, assuming a consistent level of SLS launch performance. Using the $8.4 \mathrm{~m}$ fairing drives higher system masses throughout all phases of a Mars mission, but especially for the Lander, the MAV, and the payload launch adapters as launch stacks heights and CG locations increase. Figure 20 depicts trends in initial apogee altitude and additional delta- $\mathrm{V}$ needed to insert into the target orbit. The figure shows that as the initial launch mass increases, the attainable initial apogee altitude drops steeply for small increases in mass at lower launched masses.

In order to reach the target orbit, payloads will need an additional boost. As the initial insertion orbit gets lower, more $\Delta \mathrm{V}$ will be required to reach target orbit. Options for raising payloads include:

- Use of in in-space boost stage (tug): A separatelylaunched propulsion stage rendezvous with the payload and pushes the stack to the target orbit. Requires an additional launch and adds in-space rendezvous and integration operational requirements. If the tug is intended to be multi-use, then refueling provisions and associated launches must also be considered.

- Co-launched boost stage: A 'kick' stage is integrated with the payload stack and launched together with the payloads it is pushing. The stage adds mass the launch stack, which could further reduce initial orbit altitude, unless the mass allocation for mission payloads is reduced. Depending on total mass, it may be necessary to divide the mission payloads into multiple launches.

- Increase the performance of launched propulsion systems: For payload stacks that include a transportation stage, increasing vehicle performance or loading additional propellant would allow the stage to provide the necessary boost. In addition to potential design changes, the increased propellant mass reduces mass available for other payloads.

When using solar electric propulsion for the boost, raising the payloads to the target orbit from the initial orbit can add

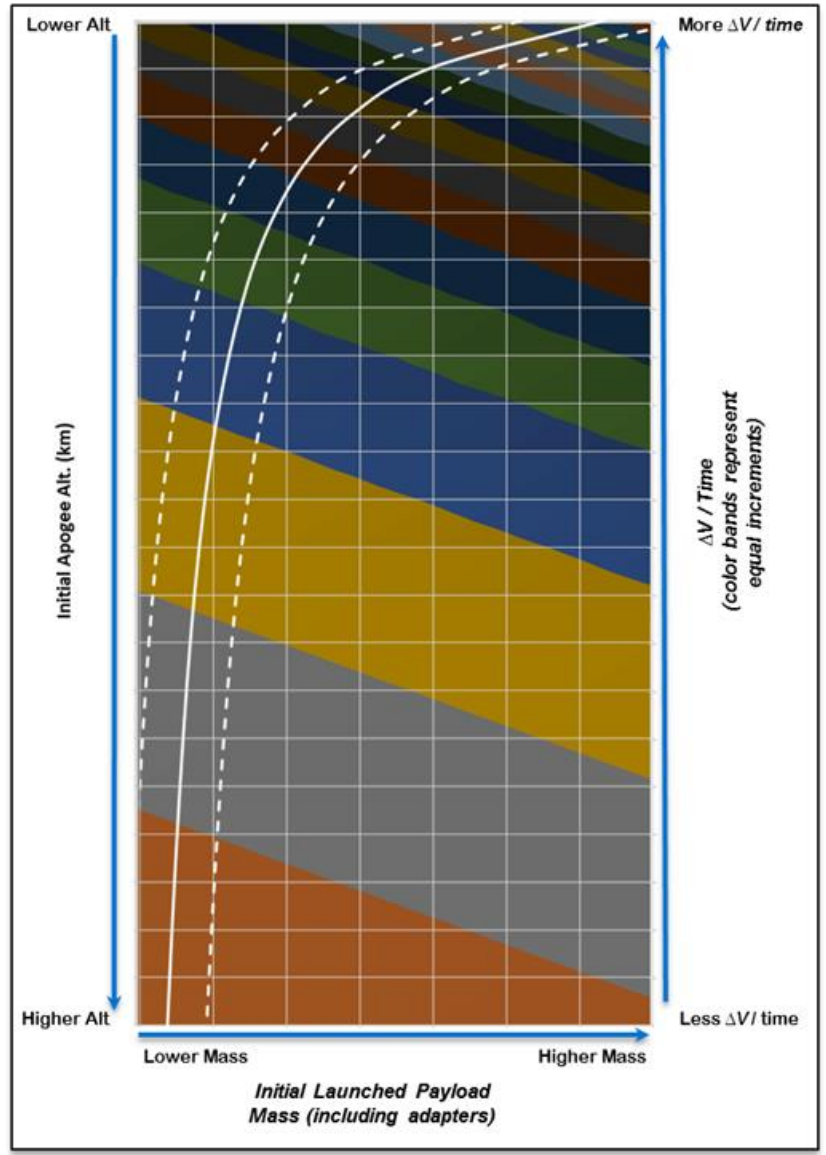

Figure 20. Initial insertion apogee altitude and 'makeup' delta-V needed to reach target orbit as initial launch mass increases.

months to years of spiral time, which in turn adds to system lifetime requirements. Longer spiral times also require elements to spend longer durations in the near-Earth thermal, radiation, and MMOD environments. This may lead to a need for increased protection, which will add more mass to the element.

Chemical stages can be used to reduce the time to reach the target orbit, however as the amount of $\Delta \mathrm{V}$ needed increases, the propellant mass required also increases, which could affect the ability to co-launch with the payloads and may incur further launch costs. Mission designers would need to trade the chemical boost stage performance and mass against launch manifest changes and other campaign impacts.

\section{Fairing height}

Based on the proposed launch manifest cases we have seen, the overall fairing height needed for EMC missions might be reduced, particularly when the $10 \mathrm{~m}$ fairing is used to launch stacks with the Mars Lander and MAV. Further analysis is needed to determine if any height reduction is feasible, and what the resultant effects on SLS would be. 


\section{Element design}

Only the Lander and MAV have design options that use the full $9.1 \mathrm{~m}$ dynamic envelope of a $10 \mathrm{~m}$ fairing. All other EMC elements were designed to fit within the $7.5 \mathrm{~m}$ dynamic envelope of the $8.4 \mathrm{~m}$ fairing, and have not been redesigned to take advantage of the larger fairing. Trends showing improvement with lower CG heights and wider diameters should show additional benefits if these elements are also redesigned to make use of maximum fairing diameter. Additional analysis is needed to determine the extent of potential improvement.

\section{KEY FINDINGS}

\section{Major Impacts of the $8.4 m$ fairing}

The driving effect of the $8.4 \mathrm{~m}$ fairing is that, all else being equal, it forces elements to be taller and narrower, and raises the element and stack CG. These changes drive element mass and performance. The combined mass of all adapters and spacecraft elements can grow by several tons to meet structural stiffness requirements of the taller, narrow stacks over comparable stacks designed for a $10 \mathrm{~m}$ fairing. For the tallest stacks the most significant increase is seen for the adapter/integration structure, but the impact on spacecraft elements cannot be discounted, including the need to redesign elements (from initial concept configurations) specifically for the launch environment.

Compounding these considerations, changes to one element can create additional effects that ripple through the architecture. The additional mass, coupled with higher CGs on the lander payload stacks affects lander performance and controllability, which increases descent propellant needs. Mass increases to the Mars mission elements lead to increased propellant needs for in-space transportation systems.

The desire to maintain launch manifests and not divide stacks into multiple launches can lead to very tall launch stacks, which significantly increases adapter mass requirements and may require integrating payloads with the fairing. The combination of adapter mass and increased element masses may drive the total launched mass of some desired launch manifests to exceed SLS performance capabilities. While there are several options for addressing this situation, all the options significantly impact the campaign, e.g., by requiring either more launches, additional boost stages, additional capabilities such as in-space refueling and assembly, lengthening the overall mission timeline, or removal of capabilities from the mission.

The volume limitations of the $8.4 \mathrm{~m}$ fairing restrict payload packaging options, and may make some design options unfeasible, as seen with the aeroentry devices. Reduced deck space on the lander affects the ability to package desired mission elements, and may prevent taking full advantage of the landed mass capacity of the lander, leaving some necessary elements for later delivery. Additionally, reduced deck space and taller payload stacks may create challenges for offloading, system deployment, and lander access, as well as adding risk for surface operations.

\section{Major Impacts of the $10 m$ fairing}

The major impacts of adopting the $10 \mathrm{~m}$ fairing, and the larger elements that take advantage of the wider diameter, are on SLS design and development schedule, availability of facilities, and ability to transport.

There are necessary design changes to the SLS to meet increased structural and performance requirements that will require upgrades to the SLS core, EUS, and Advanced Boosters. While these upgrades are part of current SLS development plans, if a Mars campaign requires earlier availability of the $10 \mathrm{~m}$ fairing the SLS development schedule and budget will be affected. These impacts will have to be weighed against impacts to the campaign implementation schedule.

If the benefits of a $10 \mathrm{~m}$ fairing are to be realized, mission elements must be designed that take advantage of the available fairing diameter. Availability of existing testing, processing, and encapsulation facilities that can accommodate 8-10m diameter payloads are limited. Without upgrading or building new facilities, complex, time-intensive testing work-arounds will be needed or else some integrated environmental tests will need to be eliminated. Investments in processing facility infrastructure are needed to safely and efficiently prepare payloads for launch. There is currently only one encapsulation facility near the launch site that is capable of accommodating $10 \mathrm{~m}$ fairings. This can affect scheduling of payload stacking operations and launch cadence.

In addition to availability of facilities that can accommodate larger elements, options for transporting large payloads around the country for testing and delivery to the Cape for launch are limited, and long-range transport is currently only available by water. Additional investments are required to provide alternative modes of transport, and to upgrade existing transportation infrastructure to handle the larger payloads. Infrastructure upgrades will also be necessary at the KSC launch site to enable transport of $10 \mathrm{~m}$ diameter launch stacks from the encapsulation facility to vehicle assembly.

\section{Minimal impacts from fairing diameter}

Regarding the impact of fairing size on EDL, three areas that were assessed; entry flight control, entry thermal flow impingement, and radiant heating, were only minimally affected by fairing diameter. Flight control requirements to meet landing accuracy offer sufficient control authority for most lander CG and stack height configurations. Any mass change for supplemental control would be negligible. Likewise, while payload stack height can increase the risk of flow impingement, the angle of attack selected for flight control adequately mitigates that risk for the payload envelop heights expected. Additionally, while analysis determined 
that lander payloads do need to be protected from radiant heating, this protection is required regardless of lander size and variances in thermal protection mass are minimal.

\section{Conclusion}

Decisions that lead to element design changes or performance reductions that increase element mass, even if seemingly small, can result in large impacts on deep space systems and missions. This is particularly evident for elements destined for the Mars surface, where the "gear ratio" (define gear ration here) associated with descending to, and ascending from the surface magnifies any mass increase and drives significantly higher launch masses.

With all other mission capabilities and design decisions being equal, fairing size selection plays a significant role in affecting Mars missions and element designs. In general, analyses have shown that shorter and wider elements and launch stacks are better for mass and performance.

Missions that do not descend to the surface of Mars can be launched in an $8.4 \mathrm{~m}$ fairing with minimal impact. For Mars surface missions, however, the decision to use an $8.4 \mathrm{~m}$ fairing can add multiple tons to a launch and affect the ability to package and manifest desired mission equipment. Accommodating these mass increases can lead to significant implications for a Mars campaign, including increased performance requirements for in-space transportation systems; the need for new capabilities such as in-space refueling and assembly; longer transit times, thus longer element lifetime requirements; and/or the need to divide payload stacks (and potentially even individual payload elements) so they can be launched on separate launch vehicles.

Using a $10 \mathrm{~m}$ fairing can mitigate many of these effects by allowing for more favorable element designs and payload configurations that are better able to meet launch and operational requirements. Adopting the $10 \mathrm{~m}$ fairing is not without costs for both SLS development and ground infrastructure, transport, and operations. These costs must be carefully considered when determining when to implement a $10 \mathrm{~m}$ fairing in exploration missions. It is clear however that maintaining a fairing-upgrade option for a $10 \mathrm{~m}$ fairing significantly reduces performance risk and increases overall mission design flexibility.

While the analyses conducted used the EMC POD architecture as the basis for analysis, the trends observed when going from wider to narrower fairings, particularly for multi-element launch stacks, are generally applicable to a wide range of missions.

\section{ACKNOWLEDGEMENTS}

The authors thank the Evolvable Mars Campaign leadership for supporting this work. The authors acknowledge the contributions of study team members Dave Paddock, John Teeter, Joshua Sams, Roger Lepsch, John Martin, Scott
Angster, David Smith, Michelle Rucker, Ronald Sostaric, Chris Cerimele, Ed Robertson, and Joseph Garcia.

\section{REFERENCES}

[1] Goodliff, K., Troutman, P., Craig, D., Caram, J., Herrmann, N., "Evolvable Mars Campaign 2016 - A Campaign Perspective," AIAA-2016-5456, 2016 AIAA Space Conference and Exposition, Long Beach, CA, Sep 2016.

[2] Rucker, M., "Large Payload Ground Transportation and Test Considerations," AIAA-2016-5624, 2016 AIAA Space Conference and Exposition, Long Beach, CA, Sep 2016.

[3] Cassell, A., Brivkalns, C., Garcia, J., Bowles, J., Kinney, D., Yount, B., McGuire, K., Wercinski, P., Cianciolo, A., Polsgrove, T. "Human Mars Mission Design Study Utilizing the Adaptive Deployable Entry and Placement Technology," IEEE Aerospace Conference, Big Sky, MT 2017 (Abstract submitted).

[4] Polsgrove, Tara P., Chapman, J., Sutherlin, S., Taylor, B., Fabisinski, L., Collins, T., Cianciolo, A., Samareh J., Robertson, E., Studak, W., Vitalpur, S., Lee, A., Rakow, G. "Human Mars Lander Design for NASA's Evolvable Mars Campaign," IEEE Aerospace Conference, Big Sky, MT, March 2016.

[5] Polsgrove, Tara P., Chapman, J., Sutherlin, S., Taylor, B., Fabisinski, L., Collins, T., Cianciolo, A., Samareh J., Robertson, E., Studak, W., Vitalpur, S., Lee, A., Rakow, G. "Human Mars Lander Design for NASA's Evolvable Mars Campaign," IEEE Aerospace Conference, Big Sky, MT, March 2016.

[6] Cerimele, C., Robertson, E., Sostaric, R., Campbell, C., Robinson, P., Hershey, M., Stachowiak, S., Matz, D., Braden, E., Johnson, B., Garcia, J., Bowles, J., Kinney, D. "A Rigid Mid Lift-to-Drag Ratio Approach to Human Mars Entry, Descent, and Landing” AIAA SciTech 2017 Grapevine TX 2017 (Abstract submitted).

[7] Dwyer Cianciolo, A. M., et al., "Entry, Descent and Landing Systems Analysis Study: Phase 1 Report," NASA-TM-2010-216720, 2010.

[8] Cianciolo, A. D., Powell, R. "Entry, Descent, and Landing Guidance and Control Apporaches to Satisfy Mars Human Mission Landing Criteria" AAS/AIAA Space Flight Mechanics Conference, San Antonio TX, Feb 2017 (Abstract Submitted). 


\section{BIOGRAPHY}

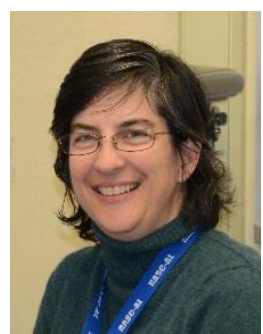

Sharon Jefferies is an aerospace engineer in the Space Mission Analysis Branch at NASA Langley Research Center. She has been at NASA since 2006 and has supported systems concept and mission designs for lunar, NEA, and Mars mission studies under the Human spaceflight Architecture Team (HAT) and for NASA's Asteroid Redirect Mission. Her background is in crew mobility and robotic systems concept development, mission concept-of-operations development, and capability needs assessment. Ms. Jefferies most recently led the integration of systems and operations across the Evolvable Mars Campaign and supported system concept development for Mars missions. Ms. Jefferies has a Bachelor of Science in Mechanical Engineering from the United States Military Academy.

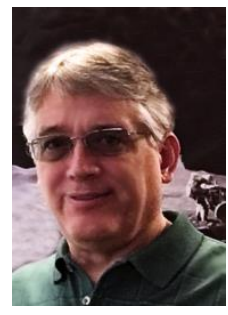

Tim Collins is an aerospace engineer in the Structural Mechanics and Concepts Branch at NASA Langley Research Center. He has been at NASA since 1987 supporting concept development and analysis of aircraft and spacecraft structural systems. Most recently he has served as the structures lead for NASA's Human-Spaceflight Architecture Team (HAT), formulating design options for future lunar and Mars missions. Mr. Collins was also the structures lead for the Altair Lunar Lander Project under NASA's former Constellation program. His prior work has included structural concepts for payload integration, aero-assisted entry systems, large space telescopes, and aircraft fuselage structures. Mr. Collins has a Bachelor's degree in Physics from the University of Rochester, and a Master of Science degree in Mechanical and Aerospace Engineering from the University of Virginia.

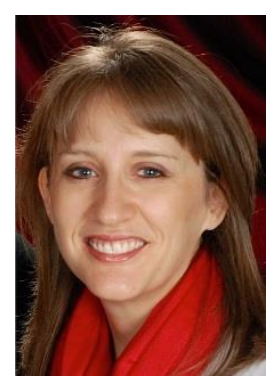

Alicia Dwyer Cianciolo is an aerospace engineer at the NASA Langley Research Center. She specializes in developing simulations to analyze vehicle flight through different atmospheres in the solar system. Primarily focusing on Mars over the past 15 years, she has worked on several missions to the planet including the Odyssey and Reconnaissance Orbiter aerobraking operations, the Exploration Rovers, and as a member of the Entry, Decent and Landing Team that successfully landed the Curiosity Rover on Mars in August of 2012. She is currently supporting NASA's the next lander mission to Mars, InSight, and is working to analyze entry technologies that will enable human exploration of the planet. She holds a Bachelor of Science degree in Physics from Creighton University and a Master of Science degree in Mechanical
Engineering from The George Washington University.

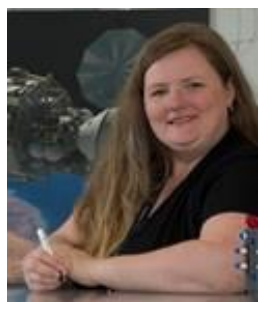

Tara Polsgrove is an aerospace engineer in the Flight Programs and Partnerships Office at NASA's Marshall Space Flight Center. She has been with NASA since 2000 and has worked on many conceptual designs of advanced spacecraft, including performance and vehicle integration for the Altair Lunar Lander. Her background is in interplanetary trajectory optimization and mission analysis. Recent work has focused on Mars transportation and lander designs supporting missions to send humans to Mars. Ms. Polsgrove has a Bachelor of Science in Aerospace Engineering from the Georgia Institute of Technology and a Master of Science in Engineering with a Systems Engineering focus from the University of Alabama in Huntsville. 
